\title{
Partial delay of reinforcement: Effects of D-length, number of different D-lengths and number of reinforcements on resistance to extinction
}

\begin{abstract}
JOHN M. COLE and JEANB. MCGOVERN, University of Portland, Portland, Ore. 97203
\end{abstract}

A test of the aftereffects hypothesis as originally applied to partial reinforcement was extended to a partial delay of reward situation. From the partial reinforcement data (Capaldi, 1964) it was predicted for the present experiment that resistance to extinction should increase with increases in (a) D-length, (b) number of different $D$-lengths, and (c) number of reinforce ments per D-length. Results of the present experiment generally confirm the predictions based on the aftereffects hypothesis.

The aftereffects hypothesis (Capaldi, 1966) suggests that reinforcement and nonreinforcement, or more accurately the organism's reaction to these events, occasion distinctive internal stimuli called stimulus aftereffects, represented as SR and $S N$, respectively. These stimulus aftereffects, though not clearly described, seem to be similar to a hypothetical stimulus trace. It has been demonstrated that the aftereffect remains functional within the organism for periods up to $24 \mathrm{~h}$ (Capaldi \& Spivey, 1964) and becomes part of the classically conditioned stimulus complex upon the occurrence of reinforcement (Capaldi \& Stanley, 1963).

The basic theoretical assumption regarding stimulus aftereffects in a partial reinforcement situation (Capaldi, 1964) is that as the $\mathrm{N}$-length (i.e., the number of successive nonreinforced trials followed by a reinforced trial) is altered, $\mathrm{SN}$ is changed quantitatively or qualitatively or both. That is, the stimulus aftereffect of a single nonreinforced trial, SN1, differs from the aftereffect of two successive nonreinforced trials, SN2, etc. Three primary variables have been used in conjunction with the aftereffects hypothesis to account for partial reinforcement effect (Capaldi, 1964). First, as the value of SN conditioned to the instrumental response is increased, (a) progressively greater values of SN receive generalized habit strength and (b) progressively greater numbers of SNs receive generalized habit strength. Second, as the number of reinforcements at a given
$\mathrm{N}$-length is increased, the tendency for the conditioned $\mathrm{SN}$ to generalize to larger $\mathrm{SN}$ values is increased. Third, several different conditioned $\mathrm{N}$-lengths produce summated generalization tendencies and these summated tendencies cause the multiple $\mathrm{N}$-length group to be more resistant to extinction than a group conditioned with a single high $\mathrm{SN}$ value.

Recently, the aftereffects hypothesis has been applied to a partial delay of reinforcement situation, wherein reinforcement is given on every trial, but is delayed on certain trials for varying amounts of time (Capaldi \& Stanley, 1963). In the partial delay of reinforcement situation, the aftereffect resulting from a delay of reinforcement (SD) is one that lies somewhere on a continuum between the aftereffect of immediate reinforcement (SR) and the aftereffect of nonreinforcement (SN). Thus, as the delay of reinforcement is increased, the aftereffect, $\mathrm{SD}$, becomes more similar to $\mathrm{SN}$ and less similar to SR.

The present experiment was designed to see if an extension of the aftereffects hypothesis as originally applied to a partial reinforcement situation can be made to predict results in a partial delay of reward situation. The design employed two delaylengths: a D60 group which received 60-sec delays on half the trials and a DV group which received delays of 5,30 , or $60 \mathrm{sec}$ on half the trials. In addition to the delay variable, three levels of acquisition were used, 24, 60, and 120 trials. Both delay and acquisition level values were arbitrarily selected as approximations to corresponding variables used in Capaldi's PR experiment (1964).

If the partial delay of reinforcement is in fact similar to the partial reinforcement situation (Capaldi, 1964) differential predictions in terms of relative resistance to extinction would be made at each level of acquisition. After 24 acquisition trials, aftereffects would predict Group D60 to be more resistant to extinction that Group DV due to increased habit strength (12 reinforcements on D60 vs four on D5, D30, and D60). After 60 acquisition trials, aftereffects would predict Group D60 to again be relatively more resistant to extinction than Group DV because of increased habit strength for the former ( 30 reinforcements on D60 vs 10 on D5, D30, and D60). After 120 acquisition trials, aftereffects would predict Group DV to be more resistant to extinction than Group D60. This switch in relative resistance to extinction is due to the fact that after 120 trials, both delay groups have attained asymptotic habit strength for all delay lengths. With equal and asymptotic habit strengths for all delay values, the aftereffects hypothesis would predict the DV group to be more resistant because the summated delay aftereffects combine or summate to produce habit strength which generalizes to more and larger values of SD in extinction.

\section{SUBJECTS}

The Ss were 42 male albino rats ranging in age from 85 to 95 days and purchased from the Holtzman Co., Madison, Wisc.

$$
\text { APPARATUS }
$$

The apparatus used was an 82-in. straight alley runway with guillotine door separating the 14-in. start section and 14-in. goal section. The alley was painted gray throughout and had a gray aluminum tube extending through the end of the goal section, making possible the remote delivery of $.45-\mathrm{mg}$ Noyes pellets into the food-cup. Alley latencies were measured by a standard timing circuit. A $10 \times 10 \times 6$ in. black box with a hinged lid served as an ITI box. PROCEDURE

Upon arrival at the lab, Ss were placed on ad lib food and water for 5 days. Prior to the initiation of acquisition trials each animal was then (a) weighed and placed on an $80 \%$ of normal body weight feeding schedule, (b) handled for 3 days, $2 \frac{1}{2}$ min per day, (c) allowed to explore the unbaited alley 5 min per day for 2 days, and (d) given five $30-\sec$ feeding trials on which the $S$ was placed by hand into the baited goal section and allowed to eat.

On each day of acquisition all groups received three immediately rewarded trials (I) and three delayed reward trials(D) daily. The sixth daily trial was always an I-trial while the first five trials were randomly ordered, D or I. The experimental variables distinguishing the six experimental groups were D-length (D60 or DV) and the total number of acquisition trials received (24, 60 , or 120 ). The D60 groups received $60 \mathrm{sec}$ of goal section delay on each designated delay trial. The DV groups received 5,30, or $60 \mathrm{sec}$ of goal section delay on designated delay trials, the order of these being randomized daily. Both number of acquisition trials and delay lengths were selected to match Capaldi (1964) and Capaldi \& Stanley (1963) studies. Food pellets were delivered via the hollow tube either immediately or after a designated delay interval, and Ss were 
allowed $15 \sec$ after delivery to consume the five pellets. Trials were terminated by removing the $S$ from the goal section and placing him in the ITI box for $20 \mathrm{sec}$ before beginning the next trial.

Extinction trials were begun on the day following a given group's last day of acquisition training. All $\mathrm{Ss}$ received 8 days of extinction, six trials per day. The procedure used during extinction was the same as that used in acquisition with two exceptions: (a) when $S$ entered the goal section on a predetermined D-trial (I and D sequence determined by the same procedure as in acquisition) the guillotine door was immediately lowered and the delay period plus $15 \mathrm{sec}$ was allowed to elapse before removal, and (b) when $S$ entered the goal section on an I trial confinement lasted for 15 sec only. During extinction Groups D60 and DV received their respective delay lengths but were never reinforced.

\section{RESULTS AND DISCUSSION}

To test for equivalence of delay groups for a given level of acquisition, $t$ tests were performed on total latencies for the first and last days of acquisition. Results indicate that delay groups within levels of acquisition did not differ significantly on any of these days.

Analysis of variance tests were performed on total extinction latencies within each level of training (start, run, and goal latencies show identical overall results and are not included). Total extinction latencies at the 24-trial level show a significant extinction effect $[F(7,84)=7.88, p<.01]$ and a significant Delay by Extinction Trials interaction $[F(7,84)=2.59, \quad p<.05]$. Inspection of Fig. 1 indicates that across extinction trials, D60 and DV groups diverged, with Group DV becoming the more resistant to extinction. This result is significant in the direction opposite that predicted by the aftereffects hypothesis.

Extinction latencies for groups at the 60-trial level show a significant extinction trials effect $[F(7,84)=4.79, p<.01]$ and a significant Delay by Extinction Trials interaction $\quad[F(7,84)=3.60, \quad p<.01]$. Inspection of Fig. 1 indicates that delay groups diverge across trials with Group D60 becoming relatively more resistant to extinction, confirming aftereffects prediction.

Latencies at the 120-trial level show a significant delay main effect $[F(1,12)=6.26, p<.05]$ and a significant extinction trials effect $[F(7,84)=7.00$, $p<.01]$. These results and inspection of Fig. 1 indicate that Group DV was more resistant to extinction than Group D60, again confirming the aftereffects predictions.

Taken as a whole, the results of the present experiment generally provide support for the aftereffects hypothesis. The

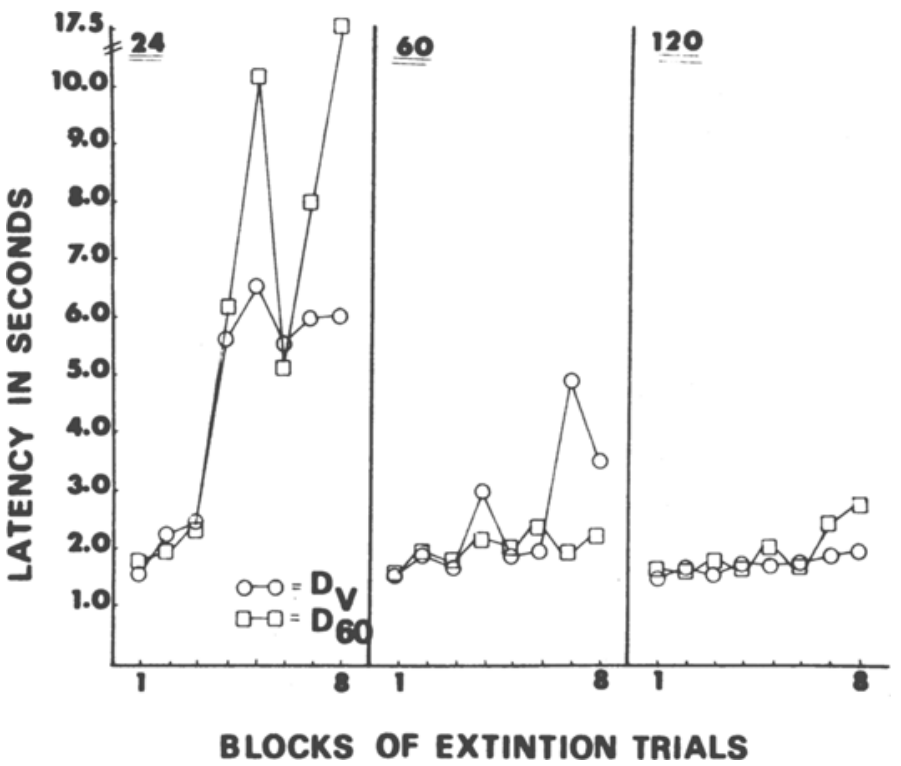

Fig. 1. Total runway latencies during extinction.

predicted switch in relative resistance to extinction occurring in the 60-and 120-trial groups can, at best, not be easily explained by other current theories. Although the data for the 24-trial groups was significant in the direction opposite to prediction, it is difficult to evaluate the importance of this result for the aftereffects hypothesis since selection of $\mathrm{N}$-length and $\mathrm{D}$-length variables was completely arbitrary. Interestingly, results at the 24-trial level and the observed switch in relative resistance to extinction from 24- to 60-trial groups cannot easily be explained by current nonsequential theories.
REFERENCES

CAPALDI, E. J. Effect of N-length, number of different $\mathbf{N}$-lengths and number of reinforcements on resistance to extinction. Journal of Experimental Psychology, 1964, 68, 230-239.

CAPALDI, E. J. Partial reinforcement: A hypothe sis of sequential effects. Psychological Review, 1966, 73, 459-477.

CAPALDI, E. J., \& SPIVEY, J. E. Intertrial reinforcement and aftereffects at 24-hour intervals. Psychonomic Science, 1964, 1, 181-182.

CAPALDI, E. J., \& STANLEY, L. R. Temporal properties of reinforcement. Journal of Experimental Psychology, 1963, 65, 169-175.

\section{The absolute behavioral taste threshold for sodium saccharin in the rat'}

JEROME S. STUMPHAUZER and RUTH ANN WIL LIAMS, Florida State University, Tallahassee, Fla. 32306

The absolute taste threshold for sodium saccharin was determined in four rats using a behavioral taste discrimination shockavoidance technique. The threshold concentrations which were obtained varied from $4 \times 10^{-5} \mathrm{M}$ to $1.2 \times 10^{-4} \mathrm{M}$, with an average value of $8 \times 10^{-5} \mathrm{M}$. As might be expected, these absolute threshold concen- 